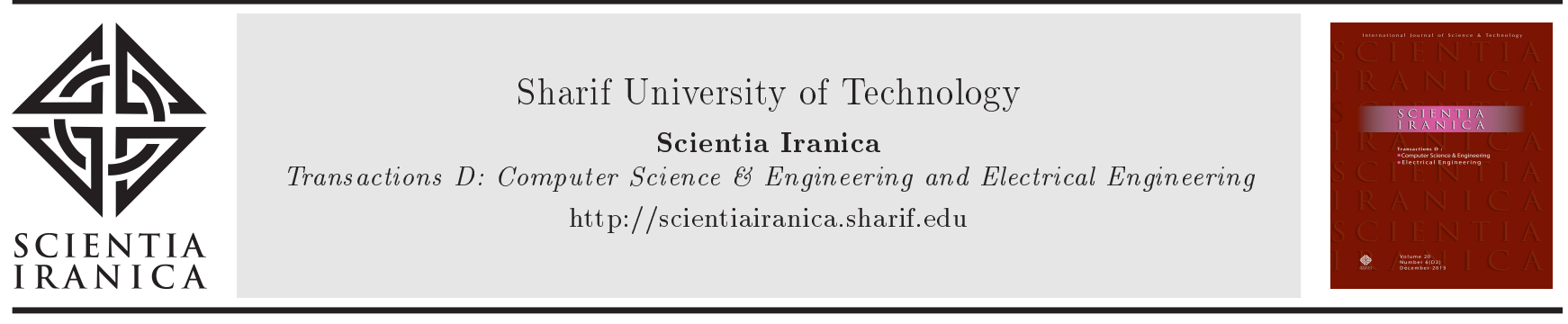

\title{
A chaotic iterative fuzzy modeling for circulating a simple sentence
}

\author{
N. Zandi-Mehran ${ }^{a}$, F. Nazarimehr ${ }^{\mathrm{a}, *}$, A.J.M. Khalaf ${ }^{\mathrm{b}}$, S. Jafari ${ }^{\mathrm{a}}$, and J.C. Sprott ${ }^{\mathrm{c}}$ \\ a. Department of Biomedical Engineering, Amirkabir University of Technology, Tehran, P.O. Box 15875-4413, Iran. \\ b. Ministry of Higher Education and Scientific Research, Baghdad, Iraq. \\ c. Department of Physics, University of Wisconsin - Madison, Madison, WI 53706, USA.
}

Received 20 January 2019; received in revised form 1 July 2019; accepted 7 September 2019

\author{
KEYWORDS \\ Sentence processor; \\ Model; \\ Chaos; \\ Fuzzy rule; \\ If-Then.
}

\begin{abstract}
The present study proposed a new model to describe possible variations of interpretation and perception of a simple sentence by different people. To illustrate the role of understandability of a simple sentence in predicting future situations, the meaning of a sentence was modeled as a fuzzy if-then rule, and the fuzzy model was investigated in an iterative process. The main objective of the paper was to model a linguistic rule using an if-then rule and its variation through one person to another. The model could predict that the interpretation reaching the final person in the following years could be chaotic and thus unpredictable.

(C) 2021 Sharif University of Technology. All rights reserved.
\end{abstract}

\section{Introduction and background}

People communicate with others through human languages. This ability helps people express their ideas and needs and enhance their skills. Advances in science are made based on transformation of information and experiments [1-3]. A sentence processor has a complex architecture. Every person views each sentence and attempts to interpret and comprehend it so as to make a new sentence with his or her individual concept. Rumor is a tale of explanations of events that circulates from one person to another, and it pertains to an object, event, or issue of public concern $[4,5]$.

An interesting tool in the modeling of expert information is the fuzzy rule which is based on fuzzy logic [6]. Fuzzy rules define the grades of membership to describe linguistic states $[7,8]$. These rules are depicted in the form of if-then rules. Fuzzy logic provides

*. Corresponding author. Tel.: +982164542398 E-mail address: fahimenazarimehr@gmail.com (F. Nazarimehr)

doi: $10.24200 /$ sci.2019.52747.2871 a method for representing fuzzy predictive modifiers including "median", "small", and "large" [9,10]. A typical fuzzy logic system consists of a knowledge base, an inference engine, and a defuzzifier [11,12]. Each fuzzy rule is in the form of if-then statements such as:

$$
\begin{aligned}
& \text { IF } x \text { is } A, \\
& \text { THEN } y \text { is } B,
\end{aligned}
$$

where $x$ and $y$ are linguistic variables and $A$ and $B$ are fuzzy membership functions. Figure 1 shows the mechanism of if-then rules in a simple example form.

Fuzzy logic is widely applicable to different fields such as nonlinear dynamics and chaos $[11,13]$. Chaos is a complex behavior with sensitive dependence on initial conditions; in other words, two initial conditions, no matter how close together, give trajectories that widely separate during the course of time [14]. Combinations of fuzzy systems and chaos have drawn much attention among researchers [15]. The generation of chaotic dynamics using fuzzy rules was studied in [16]. Modeling of chaotic dynamics using fuzzy systems and 


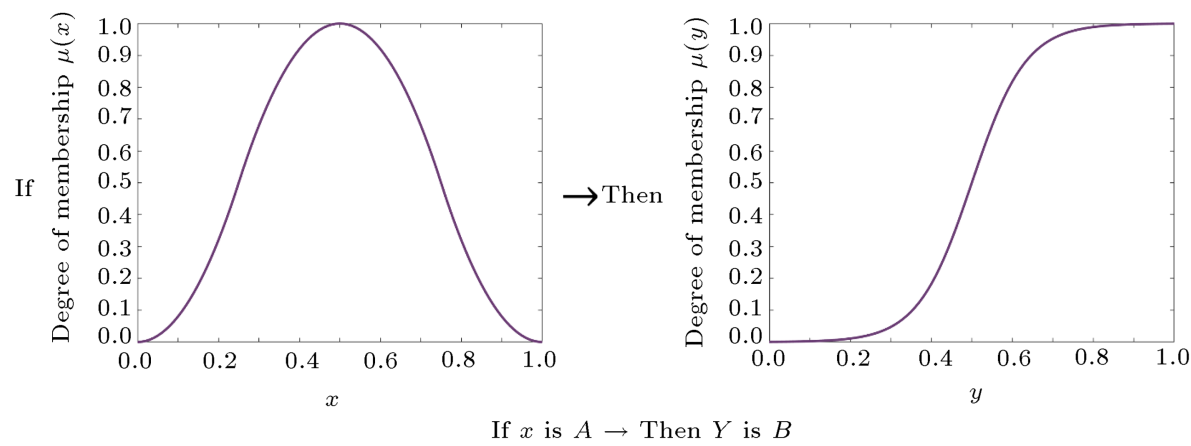

Figure 1. Mechanism of if-then rules.

linguistic description was discussed in $[17,18]$. In [19], modeling of chaotic dynamics using a small number of fuzzy rules and assigned characteristics such as Lyapunov exponents was studied. This method raises the robustness against parameter changes. Fuzzy logic is a helpful method for predicting a chaotic time series [20,21]. In [22], an adaptive fuzzy system was used to regenerate the dynamics of continuous oscillator and chaotic systems. The rules have been imposed on regions based on stretches and branches. Many studies have investigated the chaotic behavior of biological systems [23-26]. A complex Lorenz system was studied in [27]. The chaotic dynamic of visual perception was investigated in [28]. The combination of fuzzy logic and chaos seems very interesting due to its applications in the study of biological systems.

In previous studies [16-18,29], some membership functions were employed to produce a special map. However, in this paper, a linguistic rule was investigated, and iterations were added to this rule to generate interpretation of a unique sentence in the following years or its variations from one person to another. In other words, the main objective of this paper was not generating a chaotic map using fuzzy rules; instead, it aimed to model a linguistic rule. To this end, an if-then rule and its variation through one person to another were employed. Through this modeling, it was found that the interpretation results reaching the final person in the following years would be chaotic and, hence, unpredictable. In the next section, the variation of interpretation and conception of a sentence by different people is described. The fuzzy rule models this complex system in Section 3, and its different behaviors in the prediction of future situations are investigated. Finally, Section 4 concludes the study.

\section{Problem definition}

A sentence is characterized by a linear sequence of words in the language. Each natural language is a primary mode of human communications [30,31]. Functions of language include exchanging information, establishing or reinforcing social relations, and controlling the behavior of other people $[32,33]$. A sentence is interpreted by each person in the processing steps. The first step in the process of comprehending language is recognizing it from the perceptual input (spoken or written). The second step is determining how to arrange these words into sentences and then, a series of chunks of these words are analyzed [34,35].

The population of a species in biology is considered here. It is supposed that an expert says this sentence about the population: "If the population of a species in this year is normal, then its population in the next year will be large". This sentence is a simple concept that can have different meanings for different people. Qualities like "normal" and "high" can be modeled using fuzzy concepts which can imply different quantities to different people. In the next section, an individual fuzzy model of language comprehension is proposed and different predictions using this model are discussed.

\section{Fuzzy model for interpreting a language rule}

Consider the sentence "If the population of a species in this year is normal, then its population in a later year will be large". Using this sentence, each person has a forecast for the population of the next year. Different people have different interpretations of the sentence, and a fuzzy rule is an efficient method for describing the information process by these people. If we define the normalized population in one year by a variable $x$ and the normalized population in the later year by a variabley, the prediction of the population by different people during the next year can be modeled as a fuzzy if-then rule. The membership function of prediction is personal and depends on a person's social and cultural situation.

For example, imagine that the membership functions of the input and output are a bell curve (Eq. (2)) and a sigmoid function (Eq. (3)), respectively. Then, the proposed model can depict different interpretations by a person in different situations using a singleton 


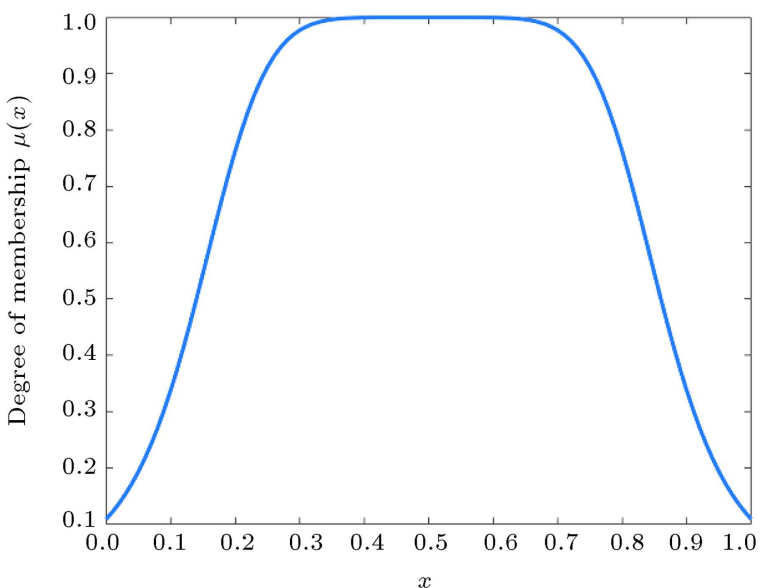

(a)

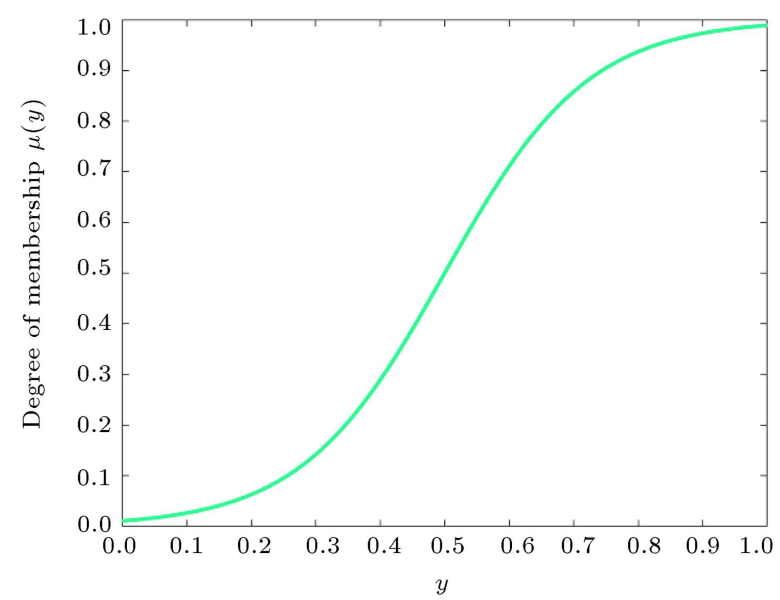

(b)

Figure 2. Input and output membership functions for one person with parameters $a_{x}=0.36, b_{x}=3.2, c_{x}=0.5$, $a_{y}=9, c_{y}=0.5$.

fuzzifier and a singleton defuzzifier.

$$
\begin{aligned}
& f\left(x ; a_{x}, b_{x}, c_{x}\right)=\frac{1}{1+\left|\frac{x-c_{x}}{a_{x}}\right|^{2 b_{x}}}, \\
& f\left(y ; a_{y}, c_{y}\right)=\frac{1}{1+e^{-a_{y}\left(y-c_{y}\right)}} .
\end{aligned}
$$

A singleton fuzzifier transforms crisp values of input into a fuzzy set with the membership function which is one for the input value and zero for other values, and a singleton defuzzifier transforms linguistic terms to crisp values [36]. Figure 2 shows an example of input and output membership functions for one person. Through such membership functions, the fuzzy model can demonstrate the process of prediction for each person one year later. Then, the person can predict the population of the following years by applying the fuzzy if-then rule (general sentence) iteratively. Figure 3 illustrates an iterative process employed in predicting

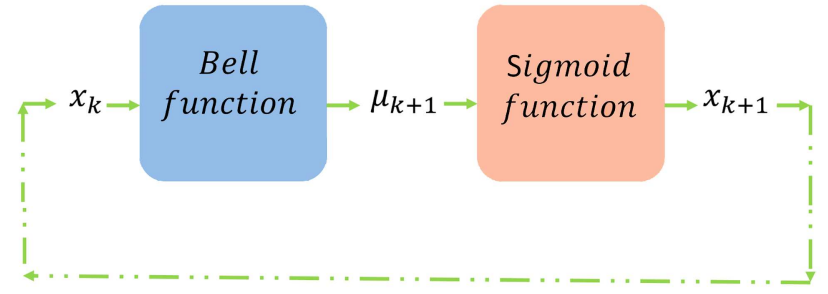

Figure 3. Prediction of the population in each year based on the population number in the previous year.

the population number each year based on the previous ones using the fuzzy model. On the contrary, the model can show different predictions of future years by different people since they have a different sentence processor and a different interpretation (as shown by different membership functions for each person in the model).

A bifurcation diagram of the model used for modifying the parameter $b_{x}$ is shown in Figure 4 . The number of iterations required to build a bifurcation diagram is 1150 , and we have removed 1000 iterations of transient time in plotting the diagram. The bifurcation parameter $b_{x}$ is a parameter of the linguistic rule representing different people's interpretations of a fuzzy modifier based on a person's social and cultural perspective. In this paper, in the assumed rule "If

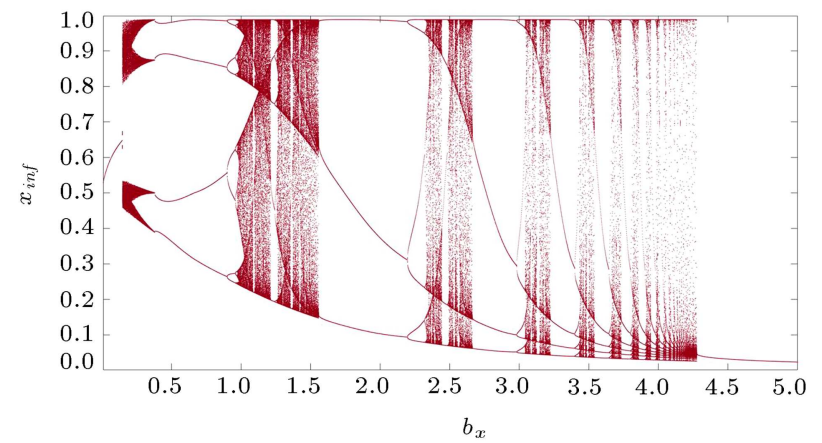

(a)

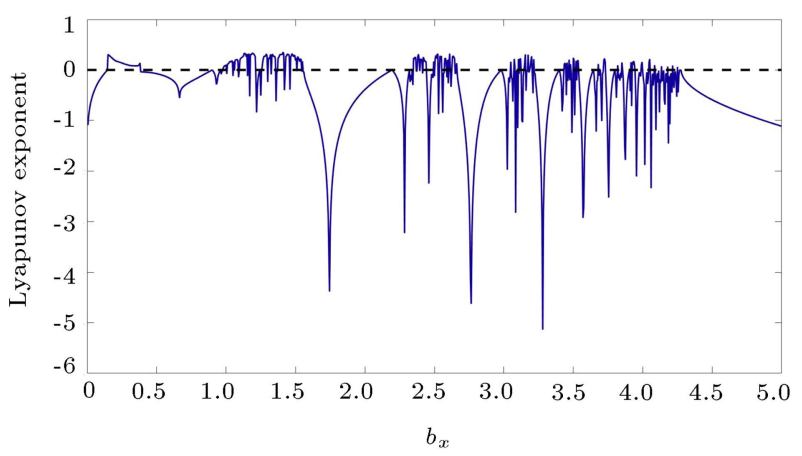

(b)

Figure 4. (a) Bifurcation diagram and (b) Lyapunov exponent of the proposed model with respect to changing personality parameter $b_{x}$ with the other parameters kept constant: $a_{x}=0.36, c_{x}=0.5, a_{y}=9, c_{y}=0.5$. 
the population of a species in this year is normal, then its population in a later year will be large", the normal modifier is represented by a bell function. The parameter $b_{x}$ in this function can change the decreasing or increasing slope. To display the modifiers "normal" and "high", different membership functions can be chosen. A sample of those membership functions was selected to describe the prediction of the population of future years by various people. Different people develop a different understanding of a sentence (different ifthen rules) and, hence, different interpretations. As an example, consider the discussed rule. Different people ascribe different meanings to this sentence depending on their personality. Changes in the personality parameter affect the prediction of the population in the later year. One person with personality parameter $b_{x}=$ 1.5 has a complex chaotic forecast for the following years, while the prediction of another person with personality parameter $b_{x}=2$ shows a less complex behavior, indicating that different people can have different forecasts of the population of future years using the same rule. A positive Lyapunov exponent in part (b) of Figure 4 confirms the chaotic predictions. In addition, Wolf's Jacobian method was employed to calculate the Lyapunov exponent in Figure 4(b) with 10000 iterations.

Here is the result of the fuzzy-related procedure in simpler algebraic words.

First, by assigning the input to a bell function,

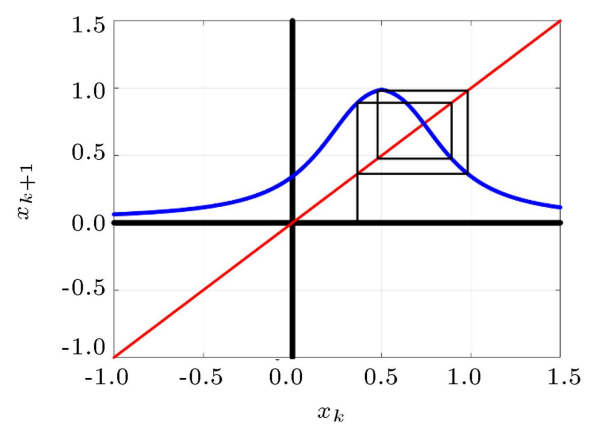

(a)

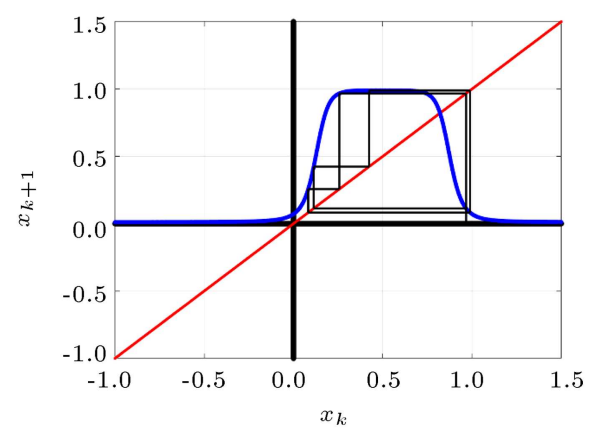

(c) fuzzification of this value (the "if" part) can be calculated. Then, this value is fed to the sigmoid function and the output is computed (the "then" part). Then, this process continues iteratively (Eq. (4)). In other words, this sentence can be considered as a onedimensional map (Eq. (5)).

$$
\begin{aligned}
x_{k+1}= & f_{2}\left(f_{1}\left(x_{k} ; a_{x}, b_{x}, c_{x}\right) ; a_{y}, c_{y}\right), \\
x_{k+1}= & \frac{1}{\left.1+e^{-a_{y}\left(\frac{1}{1+\left|\frac{x_{k}-c_{x}}{a_{x}}\right|^{2 b_{x}}}-c_{y}\right.}\right)} .
\end{aligned}
$$

Map plots and cobweb plots are two useful tools for investigating the dynamical properties of a system. To study the dynamics of System (5), a cobweb plot was used, as shown in Figure 5. The map plot of a discrete system such as $x_{k+1}=f\left(x_{k}\right)$ displays the function $f$ in its domain. The cobweb plot shows the transition of the time series (black color) within the map plot (blue) $[37,38]$. The red plot shows the identity line. Its intersection point with the map plot determines the equilibrium points. According to the map plot, System (5) has a single locally quadratic maximum. Figure 5(a) shows the cobweb plot for $b_{x}=0.5$ which has a period-4 cycle. Figure 5(b)-(d) show the cobweb plot in the chaotic, period-6, and chaotic regions, respectively. The equilibrium point of the system is

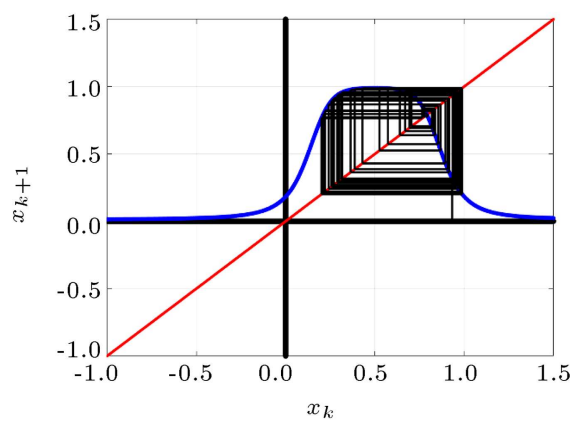

(b)

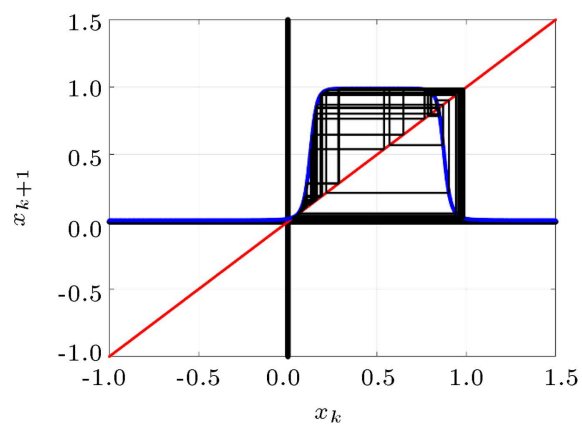

(d)

Figure 5. Cobweb plot of System (5) for (a) $b_{x}=0.5$ and initial value $x_{0}=0.36$, (b) $b_{x}=1.2$ and initial value $x_{0}=0.93$, (c) $b_{x}=2.25$ and initial value $x_{0}=0.97$, and (d) $b_{x}=4.25$ and initial value $x_{0}=0.05$. 
unstable in these cases since the absolute value of its derivative is larger than one. The equilibrium point of the system is only stable in [0.0,0.147] and [4.277, 5.0] of the studied interval, which can be seen in the bifurcation diagram of Figure 4 . The dynamics of the system always remains in the domain $[0,1]$ in the steady state. The system is characterized by a perioddoubling route to chaos at different intervals of the parameter. Moreover, there are some periodic windows in the bifurcation diagram.

This model can help linguists follow variations of interpretation of a sentence in transition between people. To establish a more precise model, its membership functions should vary for different people (iteration).

In a one-dimensional discrete dynamical system with a single locally quadratic maximum, the Feigenbaum constant has a universal value of approximately 4.66. In such a case, the Schwarzian derivative is negative [39]. The Schwarzian derivative was calculated at the studied interval of parameter $b_{x}$ which was negative according to the results. Figure 6 shows this derivative in $b_{x}=2.6$ and $b_{x}=4.5$.

Further, in a period-doubling bifurcation into

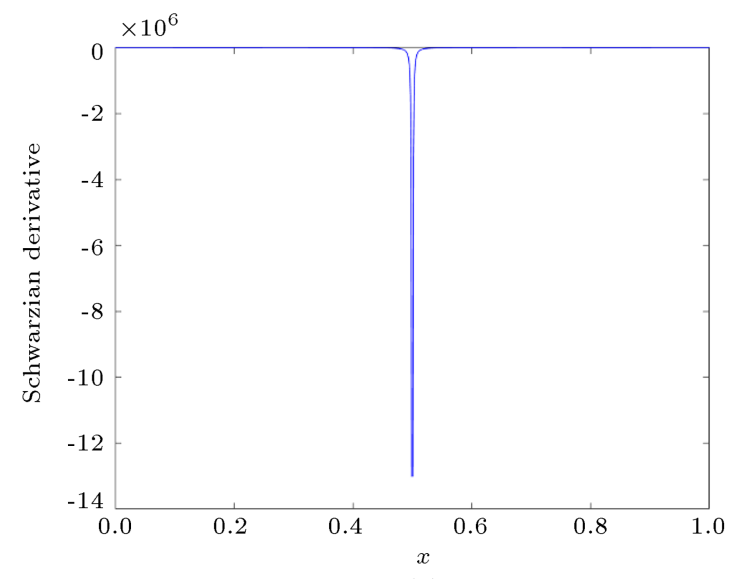

(a)

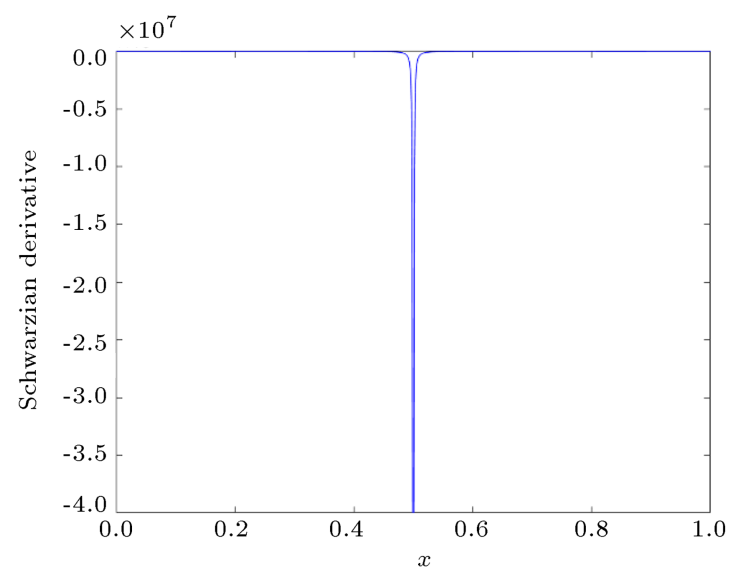

(b)

Figure 6. The Schwarzian derivative of System (5) in the range of the $x$ variable with (a) $b_{x}=2.6$ and (b) $b_{x}=4.5$. chaos, the Feigenbaum number was constant [40]. A zoomed view of the bifurcation diagram of System (5) in $b_{x} \in[0.88,1]$ is shown in Figure 7 . According to this figure, the bifurcation of period- 4 to period- 8 occurs at $b_{x}=0.900274$, the bifurcation of period- 8 to period16 at $b_{x}=0.960422$, the bifurcation of period- 16 to period-32 at $b_{x}=0.971971$, the bifurcation of period32 to period- 64 at $b_{x}=0.974364$, and the bifurcation of period-64 to period- 128 at $b_{x}=0.974870$. follows:

The Feigenbaum constant can be calculated as

$$
\delta=\lim _{n \rightarrow \infty} \frac{a_{n-1}-a_{n-2}}{a_{n}-a_{n-1}}
$$

where $a_{n}$ is the parameter for which the nth perioddoubling occurs. This constant at the studied interval is as follows:

$$
\begin{aligned}
& \delta_{1}=\frac{a_{2}-a_{1}}{a_{3}-a_{2}}=5.208069962767323, \\
& \delta_{2}=\frac{a_{3}-a_{2}}{a_{4}-a_{3}}=4.826159632260817, \\
& \delta_{3}=\frac{a_{4}-a_{3}}{a_{5}-a_{4}}=4.729249011857605 .
\end{aligned}
$$

The Feigenbaum constant approaches its limit of 4.669202 .

The proposed model can be investigated from two angles, as shown in Figure 8. First, imagine that a person uses the constant rule every year with constant parameters. Moreover, one can consider a case where people residing in a society hold the same interpretation of a sentence. In such a case, based on the bifurcation diagram of Figure 4(a), there are many parameter values for the assumed memberships $\left(b_{x}\right)$ that give chaotic dynamics. In the second point of view, by transforming the sentence from one person to another, the parameter of the membership function which determines the interpretation of one person from the sentence varies. When a person hears a sentence, he tries to understand and interpret it; then, he/she conveys his understanding of the sentence to the next person with a different membership parameter $b_{x}$. According to Figure 4(a), there are many cases in which a person's understanding of one sentence is unpredictable and chaotic.

\section{Conclusion}

The present study proposed a fuzzy model to describe variations in the prediction of future situations using a simple sentence. Application of this sentence ("If the population of a species in this year is normal, then its population in a later year will be large") to the prediction of the species population of the following 


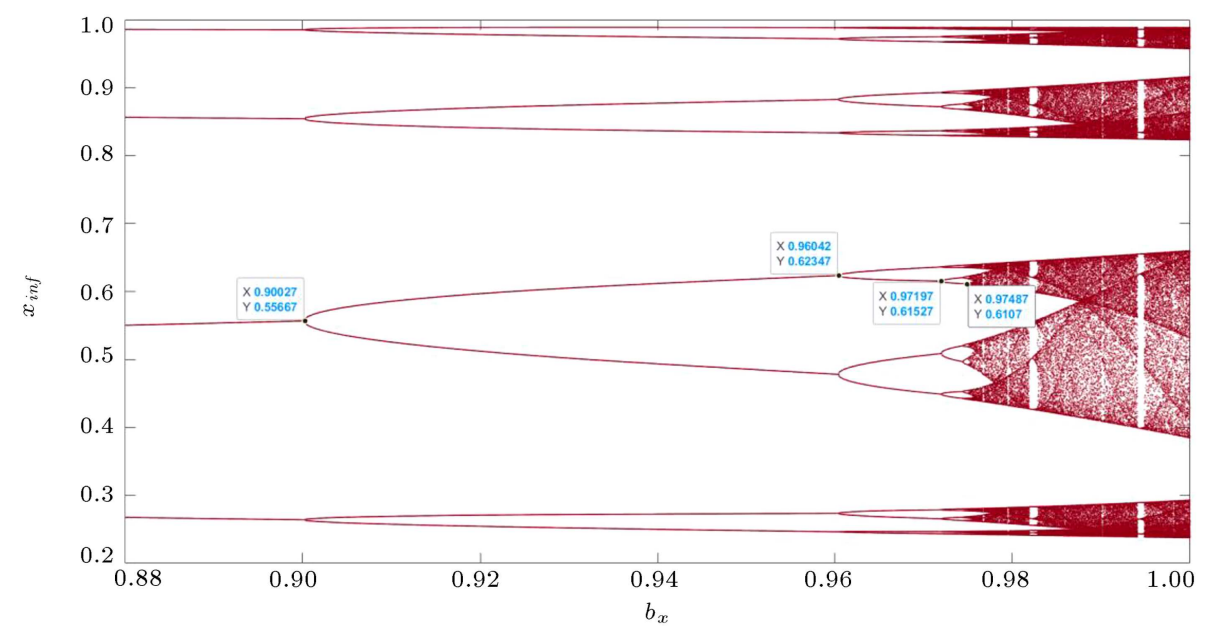

Figure 7. A zoomed view of the bifurcation diagram of System $(5)$ for $b_{x} \in[0.88,1]$.

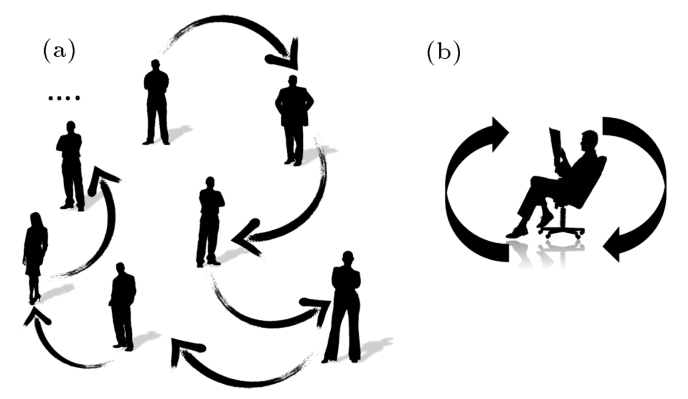

Figure 8. The two aspects of bifurcations in System (5): (a) The first aspect and (b) the second aspect.

years was investigated using a fuzzy model. Bifurcation analysis of the model showed that forecasts using the sentence could have different dynamics such as periodic and chaotic behaviors. Of note, the application of this rule did not lead to the actual population number and, yet, resulted only in the person's perception of what it might be in later years. The real population dynamics might be either complex or simple. This study also modeled the sentence interpretation and showed that the model can give chaotic dynamics. In other words, this study aimed to model a linguistic rule using an if-then rule and its variation through one person to another. Using this modeling, we found that the interpretation that passed on to the final person in the following years could be chaotic and, hence, unpredictable. This model can help linguists follow the variations of interpretation of a sentence in transition among different people. To have a more precise model, its membership functions should be altered for different people (iteration).

\section{References}

1. Schmitz, A., A Primer on Communication Studies, Retrieved September, 19, p. 2016 (2012).
2. Carlsen, W.S., "Language and science learning", in Handbook of Research on Science Education, Routledge, pp. 71-88 (2013).

3. Lindquist, K.A., MacCormack, J.K., and Shablack, H. "The role of language in emotion: Predictions from psychological constructionism", Frontiers in Psychology, 6, p. 444 (2015).

4. Peterson, W.A. and Gist, N.P. "Rumor and public opinion", American Journal of Sociology, 57(2), pp. 159-167 (1951).

5. Wang, Q., Yang, X., and Xi, W. "Effects of group arguments on rumor belief and transmission in online communities: An information cascade and group polarization perspective", Information \& Management, 55(4), pp. 441-449 (2018).

6. Guillaume, S. and Charnomordic, B. "Fuzzy inference systems: An integrated modeling environment for collaboration between expert knowledge and data using FisPro", Expert Systems with Applications, 39(10), pp. 8744-8755 (2012).

7. Zadeh, L.A. "Fuzzy sets", Information and Control, 8(3), pp. 338-353 (1965).

8. Alcantud, J.C.R. and Torra, V. "Decomposition theorems and extension principles for hesitant fuzzy sets", Information Fusion, 41, pp. 48-56 (2018).

9. Zadeh, L.A. "Fuzzy logic", Computer, IEEE, 21(4), pp. 83-93 (1988).

10. Bagheri, M., Al-jabery, K., Wunsch, D.C., et al. "A deeper look at plant uptake of environmental contaminants using intelligent approaches", Science of The Total Environment, 651, pp. 561-569 (2019).

11. Chen, L. and Chen, G. "Fuzzy modeling, prediction, and control of uncertain chaotic systems based on time series", IEEE Transactions on Circuits and Systems I: Fundamental Theory and Applications, 47(10), pp. 1527-1531 (2000). 
12. Kundu, S., Majhi, S., Karmakar, P., et al. "Augmentation of dynamical persistence in networks through asymmetric interaction", EPL (Europhysics Letters), $\mathbf{1 2 3}(3)$, p. 30001 (2018).

13. Han, L., Ding, L., and Ling-Feng, D. "Chaotic time series prediction using fuzzy sigmoid kernel-based support vector machines", Chinese Physics, 15(6), p. 1196 (2006).

14. Jafari, S., Sprott, J.C., Pham, V.-T., et al. "A new cost function for parameter estimation of chaotic systems using return maps as fingerprints", International Journal of Bifurcation and Chaos, 24(10), p. 1450134 (2014).

15. Rotshtein, A. "Integration of the fuzzy logic with chaos theory approaches in simulation and optimization of reliability", Journal of Computer and Systems Sciences International, 51(4), pp. 549-559 (2012).

16. Porto, D.M. "A fuzzy description of the Henon chaotic map", In Proceedings of the 5th WSEAS/IASME Int. Conf. on Systems Theory and Scientific Computation, pp. 15-17 (2005).

17. Porto, D.M. "Chaotic dynamics with fuzzy systems", In Integration of Fuzzy Logic and Chaos Theory, Springer, pp. 25-44 (2006).

18. Li, Z. and Halang, W.A., Integration of Fuzzy Logic and Chaos Theory, 187, Springer Science \& Business Media (2006).

19. Porto, M. and Amato, P. "A fuzzy approach for modeling chaotic dynamics with assigned properties", in Ninth IEEE International Conference on Fuzzy Systems. FUZZ-IEEE 2000 (Cat. No. 00CH37063), pp. 435-440 (2000).

20. Gentili, P.L., Gotoda, H., Dolnik, M., et al. "Analysis and prediction of aperiodic hydrodynamic oscillatory time series by feed-forward neural networks, fuzzy logic, and a local nonlinear predictor", Chaos: An Interdisciplinary Journal of Nonlinear Science, 25(1), p. 013104 (2015).

21. Nazarimehr, F., Sheikh, J., Ahmadi, M.M., et al. "Fuzzy predictive controller for chaotic flows based on continuous signals", Chaos, Solitons \& Fractals, 106, pp. 349-354 (2018).

22. Hiew, H.L. and Tsang, C.P. "An adaptive fuzzy system for modeling chaos", Information Sciences, 81(3-4), pp. 193-212 (1994).

23. Lesne, A. "Chaos in biology", In Rivista di Biologia Biology Forum, p. 467 (2006).

24. Tsumoto, K., Yoshinaga, T., Iida, H., et al. "Bifurcations in a mathematical model for circadian oscillations of clock genes", Journal of Theoretical Biology, 239(1), pp. 101-122 (2006).

25. Pham, V.-T., Vaidyanathan, S., Volos, C., et al. "A novel memristive time-delay chaotic system without equilibrium points", The European Physical Journal Special Topics, 225(1), pp. 127-136 (2016).
26. Nazarimehr, F., Jafari, S., Hashemi Golpayegani, S.M.R., et al. "Predicting tipping points of dynamical systems during a period-doubling route to chaos", Chaos: An Interdisciplinary Journal of Nonlinear Science, 28(7), p. 073102 (2018).

27. Moghtadaei, M. and Golpayegani, M.H. "Complex dynamic behaviors of the complex Lorenz system", Scientia Iranica, 19(3), pp. 733-738 (2012).

28. Beigzadeh, M. and Golpayegani, S.H. "A cellular automaton based model for visual perception based on anatomical connections", Scientia Iranica, Transaction D, Computer Science \& Engineering, Electrical, $\mathbf{2 2}(6)$, p. 2492 (2015).

29. Li, Z. "Fuzzy modeling of chaotic systems-I (Mamdani Model)", In Fuzzy Chaotic Systems, Springer, pp. 7389 (2006).

30. Mahesh, K. "Syntax semantics interactions in sentence understanding", Tecnical Report GIT-CC-95/10, College of Computing, Georgia institud of technology, Atlanta (1995).

31. Kurdi, M.Z., Natural Language Processing and Computational Linguistics: Speech, Morphology and Syntax, 1, John Wiley \& Sons (2016).

32. Valli, C. and Lucas, C., Linguistics of American Sign Language: An Introduction, Gallaudet University Press (2000).

33. Yule, G., The Study of Language, Cambridge University Press (2016).

34. Gernsbacher, M.A. and Kaschak, M.P., Language Comprehension, Wiley Online Library (2003).

35. Moreno, A., Limousin, F., Dehaene, S., et al. "Brain correlates of constituent structure in sign language comprehension", NeuroImage, 167, pp. 151-161 (2018).

36. Szczepaniak, P.S. and Lisboa, P.J., Fuzzy Systems in Medicine, 41, Physica (2012).

37. Stoop, R. and Steeb, W.-H., Berechenbares Chaos in Dynamischen Systemen, Springer-Verlag (2006).

38. Nazarimehr, F., Jafari, S., Golpayegani, S.M.R.H., et al. "Investigation of bifurcations in the process equation", International Journal of Bifurcation and Chaos, 27(13), p. 1750201 (2017).

39. Weisstein, E.W., CRC Concise Encyclopedia of Mathematics, Chapman and Hall/CRC (2002).

40. Deng, B. "Neural spike renormalization. Part IUniversal number 1", Journal of Differential Equations, 250(6), pp. 2940-2957 (2011).

\section{Biographies}

Nazanin Zandi Mehran was born in 1989 in Tehran. She received her BSc degree in Biomedical Engineering and Applied Mathematics in 2012 and her MSc degree in Biomedical Engineering in 2014 from Industrial Amirkabir University, Tehran, Iran. Currently, she is 
a PhD student in the Industrial Amirkabir University. Her main research interests include chaos theory.

Fahimeh Nazarimehr was born in 1990 in Qazvin, Iran. She received her BSc degree at Shahed University (2012), MSc degree at Sharif University of Technology (2014), and PhD at Amirkabir University of Technology, all in Biomedical Engineering. Her research interests include chaos theory and its application in biological signals and systems, nonlinear dynamics, bifurcation analysis, dynamical systems in neuroscience, bio-electricity and quantitative approaches, and feature extraction and classifcation of signals.

Abdul Jalil M. Khalaf received his BSc and MSc in Mathematics from Faculty of Science, University of Mosul, Iraq and he was awarded a PhD and Post Doctorate in Mathematics from UPM. He is a member of American Society of Mathematics, The society of Mathematical Biology, and senior member of The International Association of Computer Science and Information Technology. Dr. Khalaf was the Director of Postgraduate Studies at Kufa University and the Head of Mathematics Department at the same university.
Currently, he is an Assistant Professor at the Ministry of Higher Education and Scientific Research, Iraq. His main areas of research interest are graph theory, chemical graph theory, nonlinear dynamics, and chaos.

Sajad Jafari was born in Kermanshah, Iran in 1983. $\mathrm{He}$ received his $\mathrm{BSc}, \mathrm{MSc}$, and $\mathrm{PhD}$ degrees in Biomedical Engineering in 2005, 2008, and 2013 from Amirkabir University of Technology, Tehran, Iran. He is currently an Assistant Professor in Amirkabir University of Technology (since 2013). His research interests include artificial intelligence, optimization, pattern recognition, and especially nonlinear and chaotic signals and systems. He has published more than 100 SCI (ISI) indexed papers.

Julien Clinton Sprott received his $\mathrm{PhD}$ in Physics from the University of Wisconsin in 1969 and joined the faculty in 1973. After a 25-year career in experimental plasma physics, he became interested in computational nonlinear dynamics and chaos in 1988. He has authored or coauthored hundreds of papers on the subject and a dozen books. He is now AN Emeritus Professor of Physics at the University of Wisconsin-Madison. 